\title{
Detailed Characterization of Hospitalized COPD Patients in Relation to Combined COPD Assessment by GOLD
}

\section{Li-Cher LOH* and Choo-Khoon ONG}

PMC Lung Research, Department of Medicine, Penang Medical College, Malaysia

*Corresponding author: Li-Cher LOH, Department of Medicine, Penang Medical College, 4 Jalan Sepoy Lines, Georgetown, 10450 Penang, Malaysia, Fax: +604 228 4285; Tel: +604 228 7171; E-mail: richard_loh@pmc.edu.my

Rec date: Jan 4, 2016; Acc date: Jan 12, 2016; Pub date: Jan 19, 2016

Copyright: (c) 2016 Li-Cher LOH, et al. This is an open-access article distributed under the terms of the Creative Commons Attribution License, which permits unrestricted use, distribution, and reproduction in any medium, provided the original author and source are credited.

\section{Abstract}

Background: Detailed characterization of hospitalized COPD patients and their clinical outcomes in Malaysia is lacking. Such understanding is important to combat the rising COPD burden.

Patients and Methods: 120 patients hospitalized for COPD exacerbation in an urban-based state hospital were consecutively recruited. Comprehensive data on sociodemographics, Bristol COPD Knowledge Questionnaire (BCKQ), Hospital Anxiety and Depression Score, clinical parameters, healthcare experiences and hospital outcomes was prospectively collected from medical records and interviews with patients and/or carers.

Results: GOLD A, B, C and D categories consisted of 7 (5.8\%), $76(63.3 \%), 2(1.6 \%)$, and 35 (29.1\%) patients respectively. The mean age (SD) was $68(10.2)$ years old and $91 \%$ were male. Most had only primary school education and low incomes. Most were smokers and frequent exacerbators. About half smoked $\geq 40$ pack years and $10 \%$ had $\geq 5$ exacerbations a year. $55 \%$ had no comorbidites.

The commonest comorbidity was diabetes (20\%). $15.8 \%$ had previous TB infection. $6.6 \%$ had probably ACOS. Over half were treated with inhaled corticosteroids for $\geq 6$ months. Mean BCKQ score was $<50 \%$. Anxiety and depression were reported in $14 \%$ and $26 \%$ respectively.

Median length of hospital stay was 6 days. $11.6 \%$ had assisted ventilation. $3.3 \%$ patients died during the hospital stay. Except for Group C, distributions across categories were similar. Multivariate logistic regressions showed that the likelihoods of assisted ventilation and hospital mortality were increased with arterial $\mathrm{pCO}_{2}$ (adjusted OR 2.63, $p<0.01)$ and respiratory rate $(1.73$, $\mathrm{p}=0.01$ ) respectively. Kaplan-Meier estimates showed that GOLD categories ( $p=0.0125$ by log rank test for trend), assisted ventilation $(p=0.005)$ and antibiotic administration $(p=0.009)$ were associated with probability of prolonged hospital stay.
Conclusions: Our findings identified the many areas that can be intervened to improve COPD management. GOLD categories as a combined assessment tool of COPD severity are clinically relevant for hospitalized patients.

Keywords: COPD; Hospitalized; GOLD; Assessment; Malaysia

\section{Introduction}

Chronic obstructive pulmonary disease (COPD) is a leading global cause of morbidity and mortality that exacts substantial social and economic burden [1,2]. Its prevalence and disease burden vary across countries and people groups. The World Bank/WHO Global Burden of Disease Study has ranked COPD as the seventh most common cause of premature death in Malaysia for 2010 [3].

In collaboration with Burden of Obstructive Lung Disease (BOLD) initiative, we have recently estimated the COPD prevalence at $3.4 \%$ in a suburban population of Malaysia [4]. In a population-based survey of Asia-Pacific countries [5], over half of the interviewed COPD patients from Malaysia reported zone exacerbation in the past twelve months and many experienced negative impact on work and activities. The Malaysia figures were among the highest in the survey.

A qualitative study of local COPD patients and doctors showed a consistent theme of poor patient knowledge, compromised psycho-social and physical functioning, and lacking confidence to self-manage [6]. Although national guideline for COPD management has been available since 1999, prescribing of long-acting beta-agonist and enrolment onto pulmonary rehabilitation programme remained low [7].

The national management guideline has since been updated in 2009 [8]. Hospitalized patients with COPD studied in one local state hospital had poor quality of life with one-fifth reported exacerbations of more than twelve times a year [9].

A detailed characterization of hospitalized COPD patients and their associated hospital outcomes can provide a broad insight into how COPD is impacting local patients and a sampling of how COPD is being managed in Malaysia. 
Since 2011, Global Initiative for Chronic Obstructive Lung Disease (GOLD) has recommended a combined assessment tool to assess disease severity, health status impact and exacerbation risk in order to guide therapy [10].

We conducted a prospective study that comprehensively characterized COPD patients hospitalized for exacerbation in our local hospital and reviewed them according to the GOLD combined assessment approach.

\section{Patients and Methods}

In an observation study, all consecutive patients with COPD admitted to respiratory wards of an urban-based 1200-bed state teaching hospital were prospectively recruited over a three-month period (January to March 2013).

Included were all new or old patients diagnosed with COPD according to GOLD [10] admitted for an exacerbation. The study formed part of a larger COPD study approved by the National Research and Ethics Committee (NNMR Research no: 3998).

\section{Data collection}

Data collection was carried out using a standardized questionnaire. The data was collected by face-to-face interview (after obtaining verbal consent from patient and/or relatives) and from medical records.

They consisted of comprehensive social and demographic details (including living spouse and young children at home), known COPD risk factors (including indoor biomass burning and previous pulmonary tuberculosis), clinical details prior to admission (including previous clinic $\mathrm{FEV}_{1}$, prescribing of inhaled corticosteroids (ICS), comorbidities and COPD Assessment Tool (CAT) score [11]), breathlessness scores by modified Medical Research Council Scale (mMRC) [12], patient knowledge of COPD by Bristol COPD Knowledge Questionnaire [13], psychological status by Hospital Anxiety and Depression Score (HADS) [14], details on healthcare experience (i.e. admission hours, weekend or weekday admission), clinical parameters on arrival, doctors' interventions (i.e. antibiotic administration and assisted ventilation) and clinical outcomes (hospital death and length of hospital stay).

The diagnosis of probable ACOS is based on history of physician-diagnosed asthma and/or positive family history of asthma alone. Carlson's comorbidity index that estimates the risk of death is used to grade comorbidity severity [15].

\section{Combined COPD assessment}

Patients were categorized into Group A, B, C and D according to GOLD 2011 recommendation of a combined, multi-dimensional assessment approach [10]. The cut-off point between high and low risk of future exacerbation is based on
$\mathrm{FEV}_{1}$ of $50 \%$ predicted normal or previous reported exacerbations of three times a year.

In the latter, we chose three instead of two because of the generally high reported exacerbation rates among our cohort and that the difference between these two cut-off points is probably clinically irrelevant [16].

In our categorization, previous reported exacerbation rate is preferable to $\mathrm{FEV}_{1}$ due to missing prior $\mathrm{FEV}_{1}$ information from most patients. The cut-off point between the "more" or "less" symptoms follows the GOLD recommendation of CAT of 10 and MRC of 2 (whichever is higher).

\section{Statistical analysis}

Data is shown as GOLD categories of $A, B, C$ and D for comparison. Continuous data is described as mean and standard deviation (SD) unless otherwise specified. Categorical data is described in number and percentage.

Continuous and categorical data are analyzed by student ttests and chi-square tests respectively for differences in hospital all-cause mortality and requirement for assisted ventilation.

Variables with a $p$ value of $<0.2$ are included for simple and multivariate logistic regression analyses. Hosmer-Lemeshow test is used to ensure that the variables fit the models. KaplanMeier estimates are used to examine for associations of GOLD categories (log-rank test for trend) or other variables with length of hospital stay (log rank test). A p value of $<0.05$ is considered as statistical significant.

\section{Results}

A total of 120 patients were recruited and interviewed. Data were collected as completely as possible for all patients.

\section{GOLD categories and socio-demographic} characteristics

Majority of patients fell into Group B followed by Group D. There were only $7(5.8 \%)$ and $2(1.6 \%)$ in Group A and C respectively. The mean age was 68 years old. Most were from the age group of 60 to 79 years old. Almost all were male. While most had healthy $\mathrm{BMI}$, about a quarter to near half were either underweight or overweight in every category.

About a tenth were obese. Over two-thirds were married and lived with children and/or siblings. Less than $10 \%$ had young ( 5 years or under) children at home. Most had only primary school education. About a quarter had none or secondary education. Near $50 \%$ had income less than RM 1000 a month. The distributions of these characteristics were generally similar across all GOLD categories (Table 1).

Table 1: Patients' social and demographic characteristics. 


\begin{tabular}{|c|c|c|c|c|c|}
\hline \multicolumn{6}{|c|}{ COPD GOLD Categories } \\
\hline & A & B & C & D & Total \\
\hline $\mathrm{N}(\%)$ & $7(5.8)$ & $76(63.3)$ & $2(1.6)$ & $35(29.1)$ & $120(100)$ \\
\hline Mean age (SD), yrs & $60(8.9)$ & $68(9.5)$ & $63(3.3)$ & $68(11.7)$ & $68(10.2)$ \\
\hline \multicolumn{6}{|l|}{ Age groups } \\
\hline$<60$ yrs & $4(57.1)$ & $17(22.3)$ & $0(0)$ & $9(25.7)$ & $30(25.0)$ \\
\hline $60-79$ yrs & $3(42.8)$ & $51(67.1)$ & $2(100)$ & $19(54.2)$ & $75(62.5)$ \\
\hline$\geq 80 \mathrm{yrs}$ & $0(0)$ & $8(10.5)$ & $0(0)$ & $7(20.0)$ & $15(12.5)$ \\
\hline Male & $7(100)$ & $68(89.4)$ & $2(100)$ & $33(94.2)$ & $110(91.6)$ \\
\hline \multicolumn{6}{|l|}{ *BMI (n=82) } \\
\hline Underweight & $1(25.0)$ & $10(20.0)$ & $0(0)$ & $9(33.3)$ & $20(24.3)$ \\
\hline Healthy & $2(50.0)$ & $25(50.0)$ & $0(0)$ & $9(33.3)$ & $36(43.9)$ \\
\hline Overweight & $1(25.0)$ & $11(22.0)$ & $0(0)$ & $5(18.5)$ & $17(20.7)$ \\
\hline Obese & $0(0)$ & $4(8.0)$ & $1(100)$ & $4(14.8)$ & $9(10.9)$ \\
\hline \multicolumn{6}{|l|}{ Marital status } \\
\hline Married & $6(85.7)$ & $58(76.3)$ & $2(100)$ & $24(68.5)$ & $90(75.0)$ \\
\hline Single & $1(14.2)$ & $7(9.2)$ & $0(0)$ & $8(22.8)$ & $16(33.3)$ \\
\hline Widowed/divorced & $0(0)$ & $11(14.4)$ & $0(0)$ & $3(8.5)$ & $14(11.6)$ \\
\hline \multicolumn{6}{|l|}{ People lived with } \\
\hline Spouse only & $0(0)$ & $15(19.7)$ & $0(0)$ & $7(20.0)$ & $22(18.3)$ \\
\hline Children/siblings & $6(85.7)$ & $48(63.1)$ & $2(100)$ & $21(60.0)$ & $77(64.1)$ \\
\hline Alone/nursing home & $1(14.2)$ & $13(17.1)$ & $0(0)$ & $7(20.0)$ & $21(17.5)$ \\
\hline Having $<5$ yrs old children at home & $1(14.2)$ & $9(11.8)$ & $0(0)$ & $1(2.8)$ & $11(9.1)$ \\
\hline \multicolumn{6}{|l|}{ Education level } \\
\hline None & $1(14.2)$ & $19(25.0)$ & $0(0)$ & $10(28.5)$ & $30(25.0)$ \\
\hline Primary school & $2(28.5)$ & $34(44.7)$ & $2(100)$ & $16(45.7)$ & $54(45.0)$ \\
\hline Secondary school & $4(57.1)$ & $20(26.3)$ & $0(0)$ & $9(25.7)$ & $33(27.5)$ \\
\hline College/University & $0(0)$ & $3(3.9)$ & $0(0)$ & $0(0)$ & $3(2.5)$ \\
\hline \multicolumn{6}{|l|}{ Income per month } \\
\hline$<$ RM1000 & $1(14.2)$ & $33(43.4)$ & $1(50.0)$ & $22(62.8)$ & $57(47.5)$ \\
\hline RM1000-3000 & $5(71.4)$ & $32(42.1)$ & $1(50.0)$ & $10(28.5)$ & $48(40.0)$ \\
\hline RM3001-5000 & $1(14.2)$ & $6(7.8)$ & $0(0)$ & $0(0)$ & $7(5.8)$ \\
\hline >RM5000 & $0(0)$ & $5(6.5)$ & $0(0)$ & $3(8.5)$ & $8(6.6)$ \\
\hline
\end{tabular}

Abbreviations: COPD: Chronic Obstructive Pulmonary Disease; GOLD: Global initiative for chronic Obstructive Lung Disease; BMI: Body Mass Index; RM: Malaysian Ringgit.

Note: Figures shown are number (percentage) unless otherwise specified. *Data collected was fewer than 120.

\section{Exposure to COPD risk factors}

Majority were ex-cigarette smokers. Only $4 \%$ never smoked. Near half smoked $\geq 40$ pack years of cigarettes.
About a quarter had exposure to indoor biomass burning. The distribution was similar across GOLD B and D categories (Table 2). 
Table 2: Exposure to COPD risk factors.

\begin{tabular}{|c|c|c|c|c|c|}
\hline \multicolumn{6}{|c|}{ COPD GOLD Categories } \\
\hline & A & B & C & D & Total \\
\hline \multicolumn{6}{|l|}{ Smoking status } \\
\hline Never & $1(14.2)$ & $3(3.9)$ & $0(0)$ & $1(2.8)$ & $5(4.1)$ \\
\hline Current & $2(28.5)$ & $14(18.4)$ & $1(50.0)$ & $5(14.2)$ & $22(18.3)$ \\
\hline Ex & $4(57.1)$ & $59(77.3)$ & $1(50.0)$ & $29(82.8)$ & $93(77.5)$ \\
\hline \multicolumn{6}{|l|}{ Cigarette pack years } \\
\hline Zero & $1(14.2)$ & $3(3.9)$ & $0(0)$ & $1(2.8)$ & $5(4.1)$ \\
\hline 20-Jan & $2(28.5)$ & $6(7.8)$ & $0(0)$ & $5(14.2)$ & $13(10.8)$ \\
\hline $21-39$ & $3(42.8)$ & $23(30.2)$ & $0(0)$ & $17(48.5)$ & $43(35.8)$ \\
\hline$\geq 40$ & $1(14.2)$ & $44(57.8)$ & $2(100)$ & $12(34.2)$ & $59(49.1)$ \\
\hline Exposure to Indoor biomass burning & $3(42.8)$ & $14(18.4)$ & $0(0)$ & $10(28.5)$ & $27(22.5)$ \\
\hline \multicolumn{6}{|c|}{ Abbreviations: COPD: Chronic Obstructive Pulmonary Disease; GOLD: Global initiative for chronic Obstructive Lung Disease } \\
\hline
\end{tabular}

\section{Patients' clinical characteristics prior to} admission

Mean CAT score was 21 out of 40 . Overall mean (SD) mMRC score was $2.4(0.89)$ out of 5 . $\mathrm{FEV}_{1}$ were only available in about half the patients. The mean $\mathrm{FEV}_{1}$ (SD) was $0.9(0.30)$ litres and 42 (13.8)\% predicted normal.
About half had prior COPD exacerbations of 1 to 2 times a year. About $10 \%$ had $\geq$ five times of exacerbations a year. $13 \%$ were first-time exacerbators.

The distribution across the GOLD categories reflected these characteristics since they were delineated along these criteria (Table 3).

Table 3: Patients' clinical characteristics prior to admission.

\begin{tabular}{|c|c|c|c|c|c|}
\hline \multicolumn{6}{|c|}{ COPD GOLD Categories } \\
\hline & A & B & C & D & Total \\
\hline${ }^{*} \mathrm{CAT}(n=113)$ & $8.7(1.25)$ & $21.1(6.06)$ & $\mathrm{N} / \mathrm{A}$ & $25.1(6.10)$ & $21.8(6.71)$ \\
\hline *mMRC, $(n=115)$ & $1.6(0.81)$ & $2.2(0.78)$ & $3(0)$ & $3.0(0.83)$ & $2.4(0.89)$ \\
\hline${ }^{*} \mathrm{FEV}_{1}$ (litres) $(\mathrm{n}=64)$ & $1.2(0.39)$ & $0.9(0.26)$ & $\mathrm{N} / \mathrm{A}$ & $0.7(0.33)$ & $0.9(0.30)$ \\
\hline${ }^{*} \mathrm{FEV}_{1}(\%$ pred) $(\mathrm{n}=61)$ & $61.6(23.45)$ & $42.5(11.40)$ & $\mathrm{N} / \mathrm{A}$ & $39.9(15.64)$ & $42.7(13.80)$ \\
\hline \multicolumn{6}{|l|}{${ }^{*}$ COPD exac/yr (n=117) } \\
\hline None & $2(28.5)$ & $14(18.9)$ & $0(0)$ & $0(0)$ & $16(13.6)$ \\
\hline 2-Jan & $5(71.4)$ & $59(79.7)$ & $0(0)$ & $1(2.9)$ & $65(55.5)$ \\
\hline 4-Mar & $0(0)$ & $1(1.3)$ & $2(100)$ & $21(61.7)$ & $24(20.5)$ \\
\hline$\geq 5$ & $0(0)$ & $0(0)$ & $0(0)$ & $12(35.2)$ & $12(10.2)$ \\
\hline \multicolumn{6}{|c|}{$\begin{array}{l}\text { Abbreviations: COPD: Chronic Obstructive Pulmonary Disease; GOLD: Global initiative for chronic Obstructive Lung Disease; CAT: COPD Assessment Tool [mean } \\
\text { (SD) score]; FEV } 1 \text { : Forced Expiratory Volume in One Second [mean (SD)]; COPD exac/yr: number of COPD exacerbations in the past } 12 \text { months }\end{array}$} \\
\hline \multicolumn{6}{|c|}{ Note: Figures shown are number (percentage) unless otherwise specified. *Data collected was fewer than 120.} \\
\hline
\end{tabular}

\section{Patient's comorbidities}

Most patients had Carlson Comorbidity Index at zero. About half had no comorbidity while about a third had only one.
Overall $15.8 \%$ had previous TB infection and a fifth had diabetes mellitus. About $6.6 \%$ had probably ACOS. 
Over half had prescribed ICS over 6 months especially those in Group D. Otherwise these distributions were fairly similar across all GOLD groups (Table 4).

Table 4: Patients' comorbidities.

\begin{tabular}{|c|c|c|c|c|c|}
\hline \multicolumn{6}{|c|}{ COPD GOLD Categories } \\
\hline & A & B & C & D & Total \\
\hline \multicolumn{6}{|l|}{ Carlson $\mathrm{Cl}$} \\
\hline Zero & $5(71.4)$ & $42(55.2)$ & $1(50.0)$ & $19(54.2)$ & $67(55.8)$ \\
\hline One & $1(14.2)$ & $25(34.2)$ & $0(0)$ & $9(25.7)$ & $36(30.0)$ \\
\hline$\geq$ Two & $1(14.2)$ & $8(10.5)$ & $1(50.0)$ & $7(20.0)$ & $17(14.1)$ \\
\hline \multicolumn{6}{|c|}{ Number of comorbidity } \\
\hline None & $4(57.1)$ & $38(50.0)$ & $1(50.0)$ & $15(42.8)$ & $58(48.3)$ \\
\hline One & $3(42.8)$ & $27(35.5)$ & $0(0)$ & $13(37.1)$ & $43(35.8)$ \\
\hline Two & $0(0)$ & $9(11.8)$ & $0(0)$ & $6(17.1)$ & $15(12.5)$ \\
\hline$\geq$ Three & $0(0)$ & $2(2.6)$ & $1(50.0)$ & $1(2.8)$ & $4(3.33)$ \\
\hline Previous TB & $1(14.2)$ & $11(14.4)$ & $0(0)$ & $7(20.0)$ & $19(15.8)$ \\
\hline Diabetes & $0(0)$ & $13(17.1)$ & $1(50.0)$ & $11(31.4)$ & $25(20.8)$ \\
\hline Probable ACOS & $0(0)$ & $6(7.8)$ & $0(0)$ & $2(5.7)$ & $8(6.6)$ \\
\hline \multicolumn{6}{|c|}{ * Daily use of ICS (n=97) } \\
\hline None or $<3$ months & $3(60.0)$ & $11(18.6)$ & $0(0)$ & $2(6.4)$ & $16(16.4)$ \\
\hline 3 to 6 months & $1(20.0)$ & $11(18.6)$ & $1(50.0)$ & $6(19.3)$ & $19(19.5)$ \\
\hline 6 to 12 months & $1(20.0)$ & $21(35.5)$ & $1(50.0)$ & $5(16.1)$ & $28(28.8)$ \\
\hline$>12$ months & $0(0)$ & $16(27.1)$ & $0(0)$ & $18(58.0)$ & $34(35.0)$ \\
\hline \multicolumn{6}{|c|}{$\begin{array}{l}\text { Abbreviations: COPD: Chronic Obstructive Pulmonary Disease; GOLD: Global initiative for chronic Obstructive Lung Disease; Carlson Cl: Carlson Comorbidity } \\
\text { Index; ACOS: Asthma COPD Overlap Syndrome; ICS: Inhaled Corticosteroids }\end{array}$} \\
\hline \multicolumn{6}{|c|}{ Note: Figures shown are number (percentage) unless otherwise specified. } \\
\hline
\end{tabular}

Table 5: Bristol COPD knowledge questionnaire* .

\begin{tabular}{|c|c|c|c|c|c|}
\hline \multicolumn{6}{|c|}{ COPD GOLD Categories } \\
\hline & $A(n=6)$ & B $(n=69)$ & $C(n=1)$ & $\mathbf{D}(\mathrm{n}=32)$ & Total $(n=108)$ \\
\hline Mean scores (SD) & $23(13.0)$ & $29(11.0)$ & $30(0)$ & $26(10.0)$ & $28(10.9)$ \\
\hline Mean \% (SD) & $35(20.0)$ & $45(17.0)$ & 46 & $41(15.4)$ & $43(16.7)$ \\
\hline Low \% score & $4(66.6)$ & $45(65.2)$ & $1(100)$ & $26(81.2)$ & $76(70.3)$ \\
\hline High \% score & $2(33.3)$ & $24(34.7)$ & 0 & $6(18.7)$ & $32(29.6)$ \\
\hline \multicolumn{6}{|c|}{ Abbreviations: COPD: Chronic Obstructive Pulmonary Disease; GOLD: Global initiative for chronic Obstructive Lung Disease. } \\
\hline
\end{tabular}

\section{Bristol COPD Knowledge Questionnaire (BCKQ)}

Overall mean BCKQ score was less than 50\%. More in Group $D$ had $<50 \%$ score while more in Group A and B had $>50 \%$ score (Table 5).

This reflects an overall poor knowledge of COPD among the patients and worse in Group D (Table 5).

\section{Healthcare experiences, clinical, psychological} and laboratory parameters

Over a quarter patients were admitted during weekend and over a third during on-call hours. This reflected the emergency and unscheduled nature of their exacerbations. About a 
quarter reported depressions according to HADS while a smaller proportion (14.7\%) were anxious.

Arterial blood gases and oxygen saturation results on initial admission were fairly normal (recorded with or without oxygen supplement). Mean respiratory rate and pulse rate were 25 and 100 breaths/beats per min respectively.
Mean temperature was $37.4^{\circ} \mathrm{C}$. Mean blood pressure was in normal range. Mean blood white cell count, urea and sodium were also in normal range. All these distributions were fairly similar across all GOLD categories (Table 6).

Table 6: Healthcare experiences, clinical, psychological and laboratory parameters during initial hospital admission.

\section{COPD GOLD Categories}

\begin{tabular}{|c|c|c|c|c|c|}
\hline & A & B & C & D & Total \\
\hline \multicolumn{6}{|l|}{ Admission day, $\mathrm{n}(\%)$} \\
\hline Weekday & $4(57.1)$ & $50(65.7)$ & $2(100)$ & $30(85.7)$ & $86(71.6)$ \\
\hline Weekend & $3(42.8)$ & $26(34.2)$ & $0(0)$ & $5(14.2)$ & $34(28.3)$ \\
\hline \multicolumn{6}{|l|}{ Admission time, $\mathrm{n}(\%)$} \\
\hline Normal hrs & $2(28.5)$ & $53(69.7)$ & $1(50.0)$ & $20(57.1)$ & $76(63.3)$ \\
\hline On-call hrs & $5(71.4)$ & $23(30.2)$ & $1(50.0)$ & $15(42.8)$ & $44(36.6)$ \\
\hline \multicolumn{6}{|l|}{ *HADS, n (\%) (n=88) } \\
\hline Borderline anxiety & $0(0)$ & $10(18.5)$ & - & $9(31.0)$ & $19(21.5)$ \\
\hline Anxiety & $2(40.0)$ & $5(9.2)$ & - & $6(20.6)$ & $13(14.7)$ \\
\hline Borderline depression & $1(20.0)$ & $5(9.2)$ & - & $11(37.9)$ & $17(19.3)$ \\
\hline Depression & $0(0)$ & $13(24.0)$ & - & $10(34.4)$ & $23(26.1)$ \\
\hline \multicolumn{6}{|l|}{ Arterial blood gas } \\
\hline * $\mathrm{pH}(\mathrm{n}=113)$ & $7.34(0.55)$ & $7.36(0.07)$ & $7.31(0.13)$ & $7.36(0.10)$ & $7.36(0.08)$ \\
\hline${ }^{*} \mathrm{pO}_{2}, \mathrm{kPa}(\mathrm{n}=112)$ & $11.4(5.37)$ & $11.4(4.59)$ & $7.4(3.60)$ & $15.9(18.90)$ & $12.6(10.93)$ \\
\hline${ }^{*} \mathrm{pCO}_{2}, \mathrm{kPa}(\mathrm{n}=113)$ & $7.0(3.41)$ & $5.6(1.94)$ & $7.6(2.26)$ & $5.9(2.21)$ & $5.8(2.14)$ \\
\hline${ }^{*} \mathrm{HCO}, \mathrm{mmol} / \mathrm{L}(\mathrm{n}=112)$ & $26.6(4.76)$ & $24.7(4.90)$ & $26.4(2.61)$ & $24.3(5.52)$ & $24.7(5.03)$ \\
\hline Oxygen $\%$ saturation & $93(7.4)$ & $93(6.0)$ & $77(9.8)$ & $93(5.5)$ & $93(6.3)$ \\
\hline${ }^{*}$ Respiratory rate $(n=105)$ & $23(1.8)$ & $24(4.7)$ & $28(0)$ & $26(5.0)$ & $25(4.7)$ \\
\hline${ }^{*}$ Pulse rate $(n=119)$ & $100(13.8)$ & $98(21.4)$ & $129(26.8)$ & $103(22.6)$ & $100(21.7)$ \\
\hline Temperature ${ }^{\circ} \mathrm{C}$ & $37.2(0.83)$ & $37.4(1.20)$ & $38.0(0.35)$ & $37.4(0.48)$ & $37.4(1.01)$ \\
\hline \multicolumn{6}{|l|}{ Blood pressure, $\mathrm{mmHg}$} \\
\hline Systolic & $126(17.8)$ & $135(24.8)$ & $151(26.8)$ & $127(21.6)$ & $132(23.8)$ \\
\hline Diastolic & $81(15.8)$ & $79(13.0)$ & $89(4.2)$ & $77(13.5)$ & $79(13.5)$ \\
\hline \multicolumn{6}{|l|}{ Blood parameters } \\
\hline${ }^{*} w b c(n=115)$ & $10(5.5)$ & $13(6.0)$ & $11(3.1)$ & $14(6.1)$ & $13(6.0)$ \\
\hline *urea $(n=115)$ & $4.2(2.57)$ & $5.6(2.70)$ & $13(11.59)$ & $6.6(5.63)$ & $5.9(4.03)$ \\
\hline *sodium $(n=115)$ & $137(4.6)$ & $135(5.5)$ & $137(3.8)$ & $136(5.1)$ & $135(5.3)$ \\
\hline
\end{tabular}




\section{Interventions and clinical outcomes}

Most patients had antibiotic prescribed. Median length of hospital stay was 6 days. Assisted ventilation was needed in
$11.6 \%$ (9.1\% invasive and $2.5 \%$ non-invasive). 4 (3.3\%) patients died during the hospital stay (Table 7).

Table 7: Interventions and clinical outcomes.

\begin{tabular}{|l|l|l|l|l|l|}
\hline \multicolumn{2}{|l|}{ COPD GOLD Categories } & A & B & C & D \\
\hline *Antibiotic (n=119) & $2(28.5)$ & $62(81.5)$ & $1(100)$ & $27(77.1)$ \\
\hline LOS & 6 & 6 & 15 & 7 \\
\hline Assisted ventilation & & & $0(0)$ & $30(85.7)$ \\
\hline None & $6(85.7)$ & $70(92.1)$ & $2(100)$ & $106(88.3)$ \\
\hline Invasive & $1(14.2)$ & $4(5.2)$ & $2(2.6)$ & $0(0)$ & $11(9.1)$ \\
\hline Non-invasive & $0(0)$ & $1(1.3)$ & $0(0)$ & $3(2.5)$ \\
\hline Death & $0(0)$ & $4(3.3)$ & $3(8.5)$ \\
\hline
\end{tabular}

Abbreviations: COPD: Chronic Obstructive Pulmonary Disease; GOLD: Global initiative for chronic Obstructive Lung Disease; Antibiotic: Antibiotic Administered on Admission; LOS: Length of Hospital Stay (median days) excluding death cases.

Note: Figures shown are mean (SD) unless otherwise specified; *Data collected was fewer than 120

\section{Associations with hospital all-cause mortality and assisted ventilation}

Respiratory rate and arterial $\mathrm{pO}_{2}$ (in preference to oxygen saturation) were selected to enter into logistic regression analysis. Simple logistic regression showed that both were associated with increased likelihood of hospital mortality. Multivariate logistic regression showed that only respiratory rate was associated with increased likelihood of hospital mortality after adjusting for each other (Table 8).

Table 8: Logistic regression for hospital all-cause mortality and assisted ventilation.

\begin{tabular}{|c|c|c|c|c|}
\hline \multicolumn{5}{|l|}{ Odd Ratio $(95 \% \mathrm{Cl})$} \\
\hline & Unadjusted & $\mathbf{p}$ & Adjusted & $\mathbf{p}$ \\
\hline \multicolumn{5}{|l|}{ Hospital mortality } \\
\hline Respiratory rate & $1.53(1.15-2.03)$ & $<0.01$ & $1.73(1.09-2.74)$ & 0.01 \\
\hline Arterial $\mathrm{pO}_{2}$ & $1.42(0.96-2.03)$ & 0.05 & $1.83(0.81-4.13)$ & 0.14 \\
\hline \multicolumn{5}{|l|}{ Assisted ventilation* } \\
\hline On-call hours admission & $3.65(1.13-11.7)$ & 0.02 & $2.83(0.35-22.51)$ & 0.32 \\
\hline Arterial $\mathrm{pCO}_{2}$ & $2.65(1.74-4.04)$ & $<0.01$ & $2.63(1.48-4.65)$ & $<0.01$ \\
\hline Blood bicarbonate & $1.19(1.06-1.32)$ & $<0.01$ & $0.97(0.80-1.18)$ & 0.82 \\
\hline Respiratory rate & $1.15(1.03-1.28)$ & $<0.01$ & $1.12(0.92-1.37)$ & 0.22 \\
\hline Blood urea & $1.16(1.03-1.29)$ & $<0.01$ & $0.97(0.79-1.17)$ & 0.76 \\
\hline \multicolumn{5}{|c|}{ Abbreviations: $\mathrm{Cl}: 95 \%$ confidence interval. } \\
\hline
\end{tabular}

Admission during on-call hours, arterial $\mathrm{pCO}_{2}$ (in preference to arterial $\mathrm{pH}$ ), blood bicarbonate, respiratory rate and blood urea were selected to enter into logistic regression analysis. All showed association with increased likelihood of assisted ventilation based on simple logistic regression. After adjustment for one another, only $\mathrm{pCO}_{2}$ was associated with increased likelihood of assisted ventilation (Table 8).

\section{Association between GOLD categories with length of hospital stay}

Kaplan-Meier estimates showed that probability of prolonged hospital stay is increased with GOLD categories ( $p=0.0125$ by log rank test for trend) (Figure 1), assisted ventilation ( $p=0.005$ by log rank test) and antibiotic 
administration ( $p=0.009$ by log rank test). GOLD categories remained near statistical significant $(p=0.057)$ after adjusting for assisted ventilation and antibiotic administration

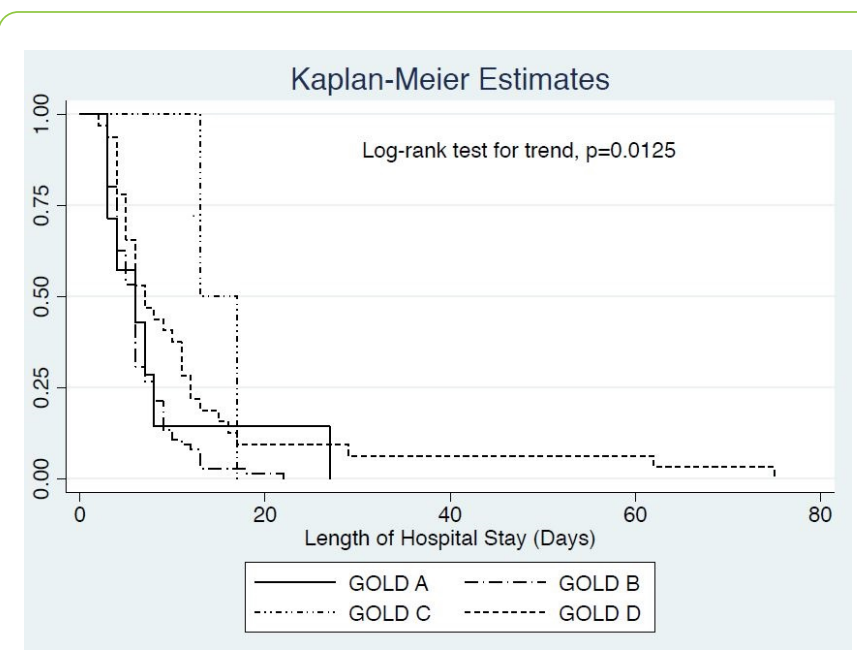

Figure 1: Kaplan-Meier estimates on probability of survival to hospital discharge by GOLD categories.

\section{Discussion}

Our findings described a wide range of illness behaviors and clinical features in hospitalized patients with COPD exacerbations. Many of these illness behaviors represented opportunities for intervention to improve COPD management. Importantly, GOLD categories appear to be clinically relevant in terms of probability of survival to hospital discharge. These GOLD categories have been introduced in 2011 guidelines as a combined, multi-dimensional assessment tool for COPD severity to guide therapy [10]. This is intuitive given that COPD has multiple symptomatic effects to contribute to its severity [17].

It is obvious that the choice of symptom measure (mMRC vs. CAT) will influence category assignment. In a Korean cohort of COPD, a CAT score of 10 was most concordant with an mMRC score of 1 , not 2 [18]. In a Spanish cohort, up to $25 \%$ of the patients could be re-categorized when these two scores were applied separately [19]. We followed the GOLD recommendation of assigning the category according to the higher score of the two systems. We based our exacerbation risk on previous exacerbation rate of 3 , not 2 because of the overall high rates of reported exacerbations among our patients. This will arbitrarily increase the threshold for Group C and $D$ and increase our patient number in Group A and B.

Consistent with other studies, Group $\mathrm{C}$ was also the least prevalent in our cohort [20-22]. In fact, our Group $C$ had only two patients and was therefore too few to allow meaningful interpretation. The larger Group B and D simply reflected our COPD cohort that was symptomatic and undergoing exacerbation. Unlike others [23], we did not observe any biological differences or differences of comorbidity prevalence in our patients. This is likely due to our smaller cohort size compared to other studies. A large observational study of
Europe and US had identified that ICS was frequently overprescribed in Group A and B patients [22]. Similarly, more than half our Group B patients were prescribed ICS for over six months. This is likely due to the common practice of indiscriminate prescribing of ICS to all diagnosed with COPD and the general availability of fixed but not separate ICS and long-acting beta-agonist inhalers.

There have been conflicting results with regards to prediction of mortality by GOLD categories [21,24]. Our findings did not support this. However we showed that GOLD categories could predict the probability of survival to hospital discharge. Antibiotic administration and assisted ventilation were two additional factors that were shown to reduce the probability of hospital discharge in our cohort. Prolonged duration of antibiotic therapy and use of specific antibiotic class have been shown to predict in-hospital treatment failure [25]. Our findings showed that the association with COPD categories remained significant even after adjusting for these two factors. To our knowledge, there has been no published study looking at the association of GOLD categories with inpatient hospital discharge. Our finding may be novel and suggest another prognostic use of the GOLD categories.

Our multivariate analyses showed that respiratory rate and arterial $\mathrm{pCO}_{2}$ were independently associated with an increased likelihood of hospital mortality and assisted ventilation respectively. These are recognized prognostic indicators of respiratory distress and failure $[26,27]$. Interestingly, arterial $\mathrm{pO}_{2}$ was associated with increased likelihood of hospital mortality from the simple logistic regression. Pre-hospital, high concentration oxygen therapy during acute exacerbation of COPD has been associated with increased mortality [28]. This is because of the potential of aggravating respiratory acidosis by inadvertently high oxygen therapy during ambulance transport. Our finding may support a need for such caution in our local setting when patients were arriving with COPD exacerbation.

Most of our hospitalized COPD patients were elderly, smokers, married and lived with children and/or siblings. Most had low income and education level. These generally reflect the socio-demographics of the patients' generation in Malaysia and the type of patients who sought treatment in government-sponsored hospital. The huge pack years of cigarette smoked and prevalence of indoor biomass exposure are also a reflection of an older age group and generation that used such means of cooking in Malaysia. The high degree of breathlessness, poor health-related quality of life and frequent exacerbations in our cohort may represent a more severe COPD disease that were not adequately addressed or treated in many of our patients. Such frequent exacerbation rates were also reported in other studies involving Malaysia [4,5]. The small number of first-time exacerbators indicates that hospitalized setting is not the ideal place to diagnose COPD early. Diabetes, and not cardiovascular disease, was the commonest comorbidity in our cohort. This observation may be due to the particular high prevalence of diabetes in Malaysia [29]. The prevalence of ACOS appears low at $6 \%$ compared to the estimated prevalence between 15 to $55 \%$ 
globally [30,31]. A poor knowledge of COPD among our COPD patients is consistent with the other Malaysian study [6] and reflects an important lack of public and patient education of COPD in Malaysia. There were a significant proportion of anxiety and depression reported in our cohort especially Group D patients. These psychological disorders are wellrecognized major comorbidities in COPD that can bring on poorer prognosis $[32,33]$. All these findings identify areas that we can intervene to improve COPD treatment in our local patient population.

\section{Acknowledgement}

The authors wish to thank the Penang Hospital Director and Director-General, Ministry of Health Malaysia for the support and approval of this research.

\section{References}

1. Lopez AD, Shibuya K, Rao C (2006) Chronic obstructive pulmonary disease: current burden and future projections. Eur Respir J 27: 397-412.

2. Mathers CD, Loncar D (2006) Projections of global mortality and burden of disease from 2002 to 2030. PLoS Med 263: 436-437.

3. Global Burden of Diseases (GBD) profile: Malaysia (2015).

4. Loh LC, Rashid A, Siti S, Gnatiuc L, Patel JH, et al. (2016) Low prevalence of obstructive lung disease in a suburban population of Malaysia: A BOLD collaborative study. Respirology.

5. Lim S, Lam DCL, Muttalif AR, Yunus F, Wongtim S, et al. (2015) Impact of chronic obstructive pulmonary disease (COPD) in the Asia-Pacific region: the EPIC Asia population-based survey. Asia Pac Fam Med 14: 4

6. Wong SSL, Abdullah N, Abdullah A, Liew SM, Ching SM, et al. (2014) Unmet needs of patients with chronic obstructive pulmonary disease (COPD): a qualitative study on patients and doctors. BMC Fam Pract 15: 67.

7. Azarisman SM, Hadzri HM, Fauzi RA, Fauzi AM, Faizal MP, et al. (2008) Compliance to national guidelines on the management of chronic obstructive pulmonary disease in Malaysia: a single centre experience. Singapore Med J 49: 886-891.

8. Malaysia National Guidelines for COPD Management (2015).

9. Loh LC, Lai CH, Liew OH, Siow YY (2005) Symptomatology and health status in patients with chronic obstructive pulmonary disease. Med J Malaysia 60: 570-577.

10. Global strategy for the diagnosis, management, and prevention of chronic obstructive pulmonary disease (Updated 2016) by Global Initiative for Chronic Obstructive Lung Disease (GOLD).

11. Jones PW, Harding G, Berry P, Wiklund I, Chen WH, et al. (2009) Development and first validation of the COPD Assessment Test. Eur Respir J 34: 648-654.

12. Celli BR, Cote CG, Marin JM, Casanova C, Montes de Oca M, et al. (2004) The body-mass index, airflow obstruction, dyspnea, and exercise capacity index in chronic obstructive pulmonary disease. N Engl J Med 350: 1005-1012.

13. White R, Walker P, Roberts S, Kalisky S, White P (2006) Bristol COPD Knowledge Questionnaire (BCKQ): Testing what we teach patients about COPD. Chron Respir Dis 3: 123-131.
14. Bjellanda I, Dahlb AA, Haugc TT, Neckelmannd D (2002) The validity of the Hospital Anxiety and Depression Scale: An updated literature review. J Psychosomatic Res 52: 69-77.

15. Charlson ME, Pompei P, Ales KL, MacKenzie CR (1987) A new method of classifying prognostic comorbidity in longitudinal studies: development and validation. J Chronic Dis 40: 373-383.

16. Schmidt $S A$, Johansen $M B$, Olsen $M, X u X$, Parker JM, et al. (2014) The impact of exacerbation frequency on mortality following acute exacerbations of COPD: a registry-based cohort study. BMJ Open 4: e006720.

17. Jones PW (2001) Health status measurement in chronic obstructive pulmonary disease. Thorax 56: 880-887.

18. Rhee CK, Kim JW, Hwang YI, Lee JH, Jung KS, et al. (2015) Discrepancies between modified Medical Research Council dyspnea score and COPD assessment test score in patients with COPD. Int J Chron Obstruct Pulmon Dis 10: 1623-1631.

19. Rieger-Reyes C, García-Tirado FJ, Rubio-Galán FJ, Marín-Trigo JM (2014) Classification of chronic obstructive pulmonary disease severity according to the new Global Initiative for Chronic Obstructive Lung Disease 2011 guidelines: COPD assessment test versus modified Medical Research Council scale. Arch Bronconeumol 50: 129-134.

20. Han MK, Mullerva H, Curran-Everett D, Dransfield DT, Washko GR, et al. (2013) GOLD 2011 disease severity classification in COPDGene: a prspective cohort study. The Lancet Respir Med 1: 43-50.

21. Soriano JB, Alfajame I, Almagro P, Casnova C, Esteban C, et al. (2012) Distribution and prognostic validity of the new GOLD grading classification. Chest 143: 694-702.

22. Vestbo J, Vogelmeier C, Small M, Higgins V (2014) Understanding the GOLD 2011 Strategy as applied to a real-world COPD population. Respir Med 108: 729-736.

23. Agusti A, Edwards LD, Celli B, Macnee W, Calverley PM, et al. (2013) Characteristics, stability and outcomes of the 2011 GOLD COPD groups in the ECLIPSE cohort. Eur Respir J 42: 636-646.

24. de Torres JP, Casanova C, Marin JM, Pinto-Plata V, Divo M, et al. (2014) Prognostic evaluation of COPD patients: GOLD 2011 versus BODE and the COPD comorbidity index COTE. Thorax 69: 799-804.

25. Cristafulli E, Torres A, Huerta A, Guerrero $M$, Gabarrus A, et al. (2015) Predicting in-hospital treatment failure ( $\leq 7$ days) in patients with COPD exacerbation using antibiotics and systemic steroids. COPD 9: 1-11.

26. Chang CL, Sullivan GD, Karalus NC, Mills GD, McLachlan JD (2011) Predicting early mortality in acute exacerbation of chronic obstructive pulmonary disease using the CURB65 score. Respirology 16: 1461-1451.

27. Yang $H$, Xiang $P$, Zhang $E$, Guo $W$, Shi $Y$, et al. (2015) Is hypercapnia associated with poor prognosis in chronic obstructive pulmonary disease? A long term follow-up cohort study. BMJ Open 15: e008909.

28. Austin MA, Wills KE, Blizzard L, Walters EH, Wood-Baker R (2010) Effect of high flow oxygen on mortality in chronic obstructive pulmonary disease patients in prehospital setting: randomized controlled trial. BMJ 341: c5462.

29. Ramachandran A, Snehalatha C, Shetty AS, Nanditha A (2012) Trends in prevalence of diabetes in Asian countries. World J Diabetes 15: 110-117. 
30. Marsh SE, Travers J, Weatherall M (2008) Proportional classi cations of COPD phenotypes. Thorax 63: 761-767.

31. Weatherall M, Travers J, Shirtcliffe PM (2009) Distinct clinical phenotypes of airways disease de ned by cluster analysis. Eur Respir J 34: 812-818.

32. Hanania NA, Mullerova H, Locantore NW (2011) Determinants of depression in the ECLIPSE chornic obstructive pulmonary disease cohort. Am J Respir Crit Care Med 183: 604-611.
33. Ng TP, Niti M, Tan WC, Cao Z, Ong KC, et al. (2007) Depressie symptoms and chronic obstructive pulmonary disease: effect on mortality, hospital readmission, symptom burden, functional status, and quality of life. Arch Intern Med 167: 60-67. 